\title{
The Use of Artificial Intelligence Combined With Cloud Computing in the Design of Education Information Management Platform
}

\author{
https://doi.org/10.3991/ijet.v16i05.20309 \\ Qiang Li \\ Zhengzhou University of Aeronautics, Zhengzhou, China \\ levisBJe163. com
}

\begin{abstract}
Based on cloud computing theory and service-oriented architecture (SOA) design pattern, a smart education management platform is designed by using cloud computing and artificial intelligence technology. The platform is deployed in the server cluster environment, with Hadpoop managed storage cluster as the data storage center. For the education management, education portal and remote classroom, the corresponding services are launched, which has the advantages of intelligent and efficient, massive data access and intelligent collaborative management. The test results show that the platform can be used normally in the ports of computer, mobile phone and tablet computer, and can successfully complete the basic operations such as user registration and login, educational administration, and content storage. The results of database stress test show that the total time consumption of sequential read, sequential write, random read and random write is $752 \mathrm{~s}, 312 \mathrm{~s}, 968 \mathrm{~s}$ and $211 \mathrm{~s}$, respectively when millions of simulated data are inserted into the database, which indicates that the database can support large-scale data access. The stress test results of content storage service show that when the number of clients is adjusted to 500, 1000 and 3000 , the output quantity of each read-write interface can be maintained at about $3500 \mathrm{pages} / \mathrm{min}$ in $60 \mathrm{~s}$, indicating that the system can still run stably under the condition of high concurrency. The management platform discussed has practical significance to promote the development of intelligent and information-based education management.
\end{abstract}

Keywords - Artificial intelligence; cloud computing; education information management; data storage

\section{Introduction}

With the rapid development of society and the continuous improvement of education level, the number of college students in China has increased rapidly. According to the National Education Development Statistical Bulletin In 2019 issued by the ministry of education, there were 2688 ordinary colleges and universities in China, with a total number of 40,020,000 students in various types of higher education school, and the enrollment of college students had increased compared with 
that of the previous year. Under the actual conditions, how to do a good job in the education, management and service of students and teachers has become the core issue to be considered, and the success of education management service is also the key to the development of a school.

As a booming intelligent computing technology, cloud computing can complete the efficient processing of large-scale data in seconds, greatly improving the computing time and efficiency [1]. Cloud computing technology is widely used in all walks of life because of its powerful computing function, and good application effect has also been achieved in education management [2]. Wen et al. [3] fully explained the advantages and future development prospects of applying cloud computing to the construction of higher education management informatization. Sabi et al. [4] designed a set of intelligent management model for sub-Saharan Africa University based on cloud computing technology, and analyzed its generation systematically. Liu et al. [5] proposed a cloud platform scheme of university teaching integrated management based on cloud computing, which achieves good results in practical application. It can be concluded that in the era of rapid development of artificial intelligence (AI), the application of cloud computing technology in education management has not only achieved good application results, but also has broad development prospects.

The current era background is fully considered. An intelligent education platform based on AI and cloud computing technology is designed, which realizes two core functional areas of intelligent education service system and intelligent education application system, and realizes the orderly operation of three main functional modules: educational resource storage, remote classroom function and educational administration management. Moreover, the test results show that the system can meet the requirements of high concurrency and big data processing. The research content can greatly improve the efficiency of school management, and provide scientific and effective reference materials for the optimization management and intelligent development of school education management in the future.

\section{$2 \quad$ Method}

\subsection{Analysis of the functional requirements of education management platform}

In order to meet the actual needs of the current education management field, the education management platform designed must adapt to the growing number of students, meet the gradually diversified learning needs and pursue a more efficient and intelligent management mode. Therefore, in the construction of intelligent management platform, the following functional modules are mainly designed, as shown in Table 1. The function modules of intelligent education platform are mainly divided into intelligent education service module and intelligent education application module. Among them, the intelligent education service module can complete the user registration and management, service information registration, search, management and other functions. Intelligent education application module is the most important 
part of this system and the main functions of the system are completed in this module, including educational resource storage, remote classroom function and educational administration management, which are introduced in detail as follows.

Table 1. Intelligent education platform function module

\begin{tabular}{|l|l|}
\hline \multicolumn{1}{|c|}{ Classification } & \multicolumn{1}{c|}{ Function } \\
\hline \multirow{4}{*}{ Intelligent education service module } & User registration \\
\cline { 2 - 2 } & User management \\
\cline { 2 - 2 } Intelligent education application module & Service registration \\
\cline { 2 - 2 } & Service lookup \\
\cline { 2 - 2 } & Service management \\
\hline & Educational resource storage \\
\cline { 2 - 2 } & Remote classroom function \\
\cline { 2 - 2 } & Educational administration management \\
\hline
\end{tabular}

Educational resource storage: With the increasing popularity of the concepts of "lifelong learning" and "all-round development", people's demand for all kinds of knowledge is gradually deepening. Moreover, for a user who receives a lot of knowledge, a platform that can store and manage in real time will undoubtedly save a lot of time [6]. Therefore, in the design of intelligent education platform, the intelligent storage of educational resources should be considered at first.

Figure 1 shows the functional requirements of the intelligent education platform. The educational resource storage service needs to meet the user's download, upload, expansion and management requirements for massive educational resources [7]. At the same time, it can meet the user's operational requirements such as classification and sorting of resource data, support data transfer between users, and realize the sharing of data resources. For managers, this function can meet the administrator's background management operation, and realize the user's basic information query, management, maintenance.

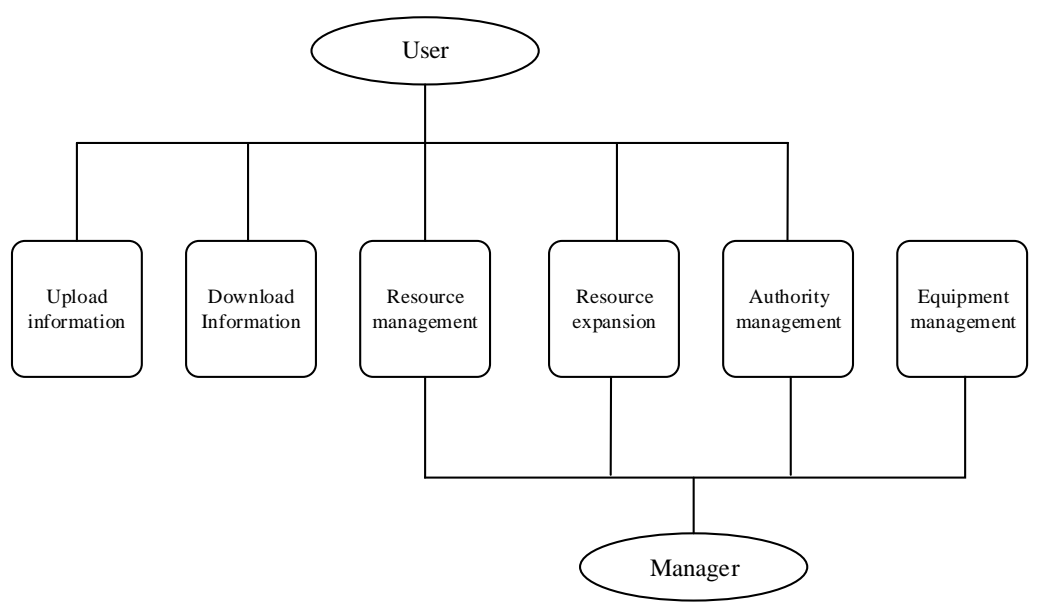

Fig. 1. Educational resource storage function requirements 
Remote classroom function: Remote classroom function can meet the needs of students' learning at any time, and has become the most important teaching method in some cases where there is no field class [8]. Especially in the outbreak of corona virus disease, the remote classroom function plays a huge role, and becomes an important way for teachers to develop normal teaching. This requires the education platform to meet the needs of students for remote education. As shown in Figure 2, the education management platform studied can meet the needs of students in remote class, online examination and communication interaction, and meet a series of operations such as classroom management, classroom interaction, examination supervision of teachers. Students and teachers can use this platform to have a good and smooth remote interactive communication and improve the efficiency of remote learning.

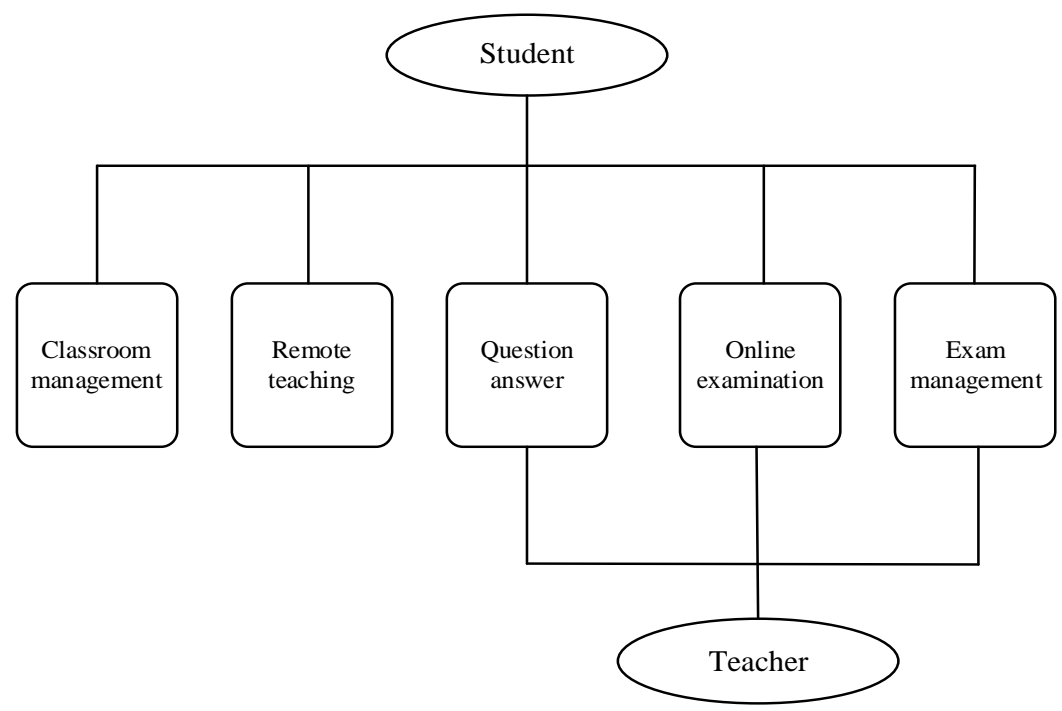

Fig. 2. Functional requirements of remote classroom

Educational administration management: With the increasing number of students in school, the wide application of intelligent educational administration management function can not only further improve the efficiency of daily management of students, but also reduce the work pressure of school administrators and greatly save the time of teachers and students [9]. As shown in Figure 3, students use the platform to manage data, select courses, and query scores. Teachers can use the platform to complete the course management, score input, performance management and other work. The school administrators can arrange courses, manage the data of teachers and students, and count scores by using this function, which greatly saves working time and improves the work efficiency. 


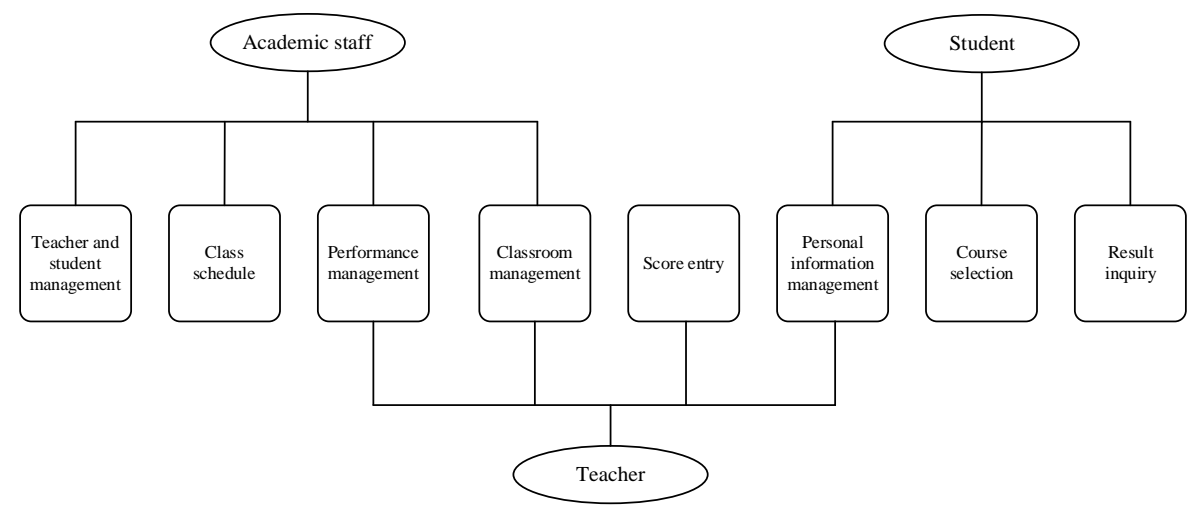

Fig. 3. Educational administration management function requirements

\subsection{Design of intelligent education platform architecture based on cloud computing}

Intelligent education platform is mainly divided into three parts, which are bottom facilities layer, system construction layer and application experiment layer from bottom to top. The bottom facilities layer is the basic part of the whole platform operation, which includes not only a series of basic hardware facilities such as large capacity storage, computer and high-speed network, but also the virtual operating environment such as Hadoop distributed framework, MySQL database and Web server.

The system construction layer is the core system of platform function test. In this layer, the main platform functions are constructed, such as educational resource storage, remote classroom function and educational administration management function, and a management part is also established, which can monitor and maintain the various functional modules in the platform in real time. The main objects of educational resource storage function are ordinary users and platform administrators. Ordinary users are students, teachers and other registered users who can use the function to upload, download and share the educational resources they need. The platform administrator is responsible for the whole module monitoring management and resource allocation, setting storage space and other operations. The collected educational resources are stored in Hadoop storage cluster. Compared with the traditional storage method, it has the advantages of reliable storage, economical price, convenient expansion, stable load and flexible sharing [10]. In the remote classroom function module, there are two parts: online teaching and online examination. The main objects are teachers and students. After the teacher logs in to the system, the courseware is displayed to explain the course, and the students listen to the lecture at the other end of the computer. At the same time, the content can be played back after class, so that students can further understand the content. The main audience of educational administration management module is school administrators, teachers and students. Using this module, school administrators can flexibly arrange the course and 
examination time according to the teacher's arrangement and students' class time; students can select courses, query results and take other operations; teachers can further optimize the teaching content and progress according to the examination results.

The application layer of the system is mainly based on the front-end development technology, which connects the lower education resources and service interfaces, and provides rich application services for users. Moreover, it will verify the identity according to the feedback of different users, and provide corresponding services for them.

\subsection{Key technologies of the realization of information management system}

Service-Oriented Architecture (SOA): SOA is a general architecture, which splits different functional units (also called services) in a certain environment, and finally provides these services to other customers $[11,12]$. Some researchers believe that the so-called service is a function that is precisely defined, packaged and independent of the environment and pile of other services, and it is also a collection of services in essence [13]. Web services standards are the core of SOA. According to this standard, SOA further standardizes its services, so that the services provided by SOA can be used on computers, mobile phones, tablets and other terminals [14]. Compared with the traditional software architecture, the development and maintenance of SOA design architecture is more convenient, and its internal organization is very flexible. When a function requirement changes, the designer only needs to adjust the function flow inside the service, and there is no need to repair the service interface, which greatly saves time and operation cost.

Hadoop architecture: Hadoop architecture is an open-source architecture widely used in cloud computing technology, which can effectively improve the computing and storage capability [15]. MapReduce which can handle large-scale data and Hadoop distributed file system (HDFS) which has distributed data storage capability, are two core technologies [16]. MapReduce is a distributed processing computing model, which can provide the core algorithm for Hadoop architecture, and enable Hadoop architecture to realize complex workflow such as work scheduling, data partition, summary analysis, and fault tolerance mechanism [17]. When it is used, the developer can implement the function by simply writing map method and reduce method. HDFS is a large distributed file system, which can build an efficient and stable storage cluster on personal computer. The storage mode of HDFS is partitioned storage, that is, files are partitioned into different files [18]. In addition to the above two core technologies, Hadoop architecture also includes Hive and HBase and other structures. Hive is a data warehouse structure based on HDFS, while HBase is a large and efficient distributed storage database $[19,20]$. 


\subsection{Test and development environment of platform system}

In order to test the running effect of the platform system, the system is developed and tested in the small virtual machine environment. Table 2 shows the main development environments.

Table 2. Platform development environment

\begin{tabular}{|l|l|l|l|}
\hline \multicolumn{1}{|c|}{ Software } & \multicolumn{1}{|c|}{ Comment } & Software & \multicolumn{1}{c|}{ Comment } \\
\hline Windows 10 & $\begin{array}{l}\text { Operating system for development } \\
\text { environment }\end{array}$ & Eclipse & $\begin{array}{l}\text { Development platform, code development, } \\
\text { compilation and debugging }\end{array}$ \\
\hline Nginx & Load balance & Tomcat & Web Server \\
\hline Hadoop & $\begin{array}{l}\text { Distributed computing and storage } \\
\text { environment }\end{array}$ & Hbase & Basic database \\
\hline MySQL & Extended database & VMware & Virtual machine environment \\
\hline CentOS & Virtual machine operating system & JDK & Java Development Kit \\
\hline
\end{tabular}

The test environment is mainly composed of one physical machine and six virtual machines. One physical machine is used to simulate the client sending various service requests. Among the six virtual machines, two are servers, which are used to deploy the operating environment and function code of the platform, and the remaining four are deployed as the resource storage function in the media of Hadoop storage platform.

\section{$3 \quad$ Results and Discussion}

\subsection{Analysis of the influence of large volume data on the operation of information management database}

Database is the core function of the platform, and the stability of its operation directly determines the use of the platform. Stress test is carried out on the database. Four indexes of sequential read, sequential write, random read and random write in the database are tested under the insertion of millions of simulated data. Figure 4 shows the detailed results. The stress test results of the database show that when the million-level data is inserted, the total time consumption of sequential read in the database is $752 \mathrm{~s}$, with the rate of $1330 \mathrm{row} / \mathrm{s}$; the total time consumption of sequential write in the database is $312 \mathrm{~s}$, with the rate of $3205 \mathrm{row} / \mathrm{s}$; the total time consumption of random read in the database is $968 \mathrm{~s}$, with the rate of $1033 \mathrm{row} / \mathrm{s}$; the total time consumption of random write in the database is $211 \mathrm{~s}$, with the rate of 4739 row/s. The experimental results show that when large-scale data is inserted, the platform database runs well and good results are achieved in the data rate pressure test. 


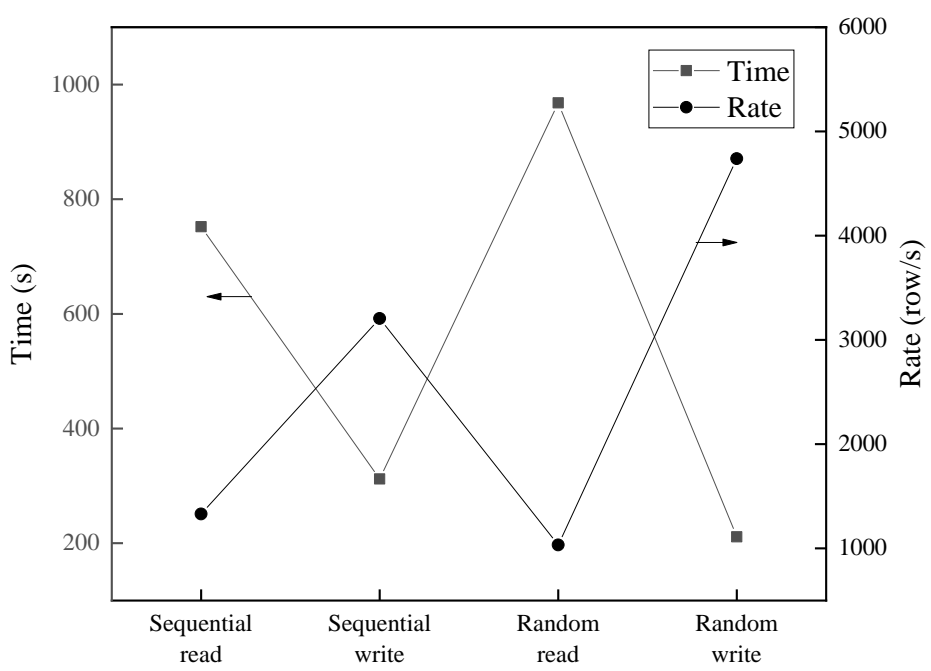

Fig. 4. Database stress test chart

\subsection{Analysis on the operation of education information management platform in high concurrency state}

Due to the large number of school staff, in some special time, there may be too many logins and more service requests, which may lead to instability of the platform system due to excessive load. In order to prevent this situation, the load capacity of the platform is tested. The read-write interface is taken as an example. The service request is sent to the platform when the number of clients is 500, 1000 and 3000 in $60 \mathrm{~s}$, and the average number of service outputs per minute is studied. Figure 5 shows the test results. When the number of clients is 500 , the average rate of service read interface is $3560 \mathrm{pages} / \mathrm{min}$ and that of write interface is $3752 \mathrm{pages} / \mathrm{min}$; when the number of clients is 1000 , the average rate of service read interface is 3334 pages/min, and that of write interface is $3518 \mathrm{pages} / \mathrm{min}$; when the number of clients is 3000 , the average rate of service read interface is $3211 \mathrm{pages} / \mathrm{min}$, and that of write interface is $3598 \mathrm{pages} / \mathrm{min}$. The experimental data show that the platform can still run stably and smoothly in the case of high concurrency. 


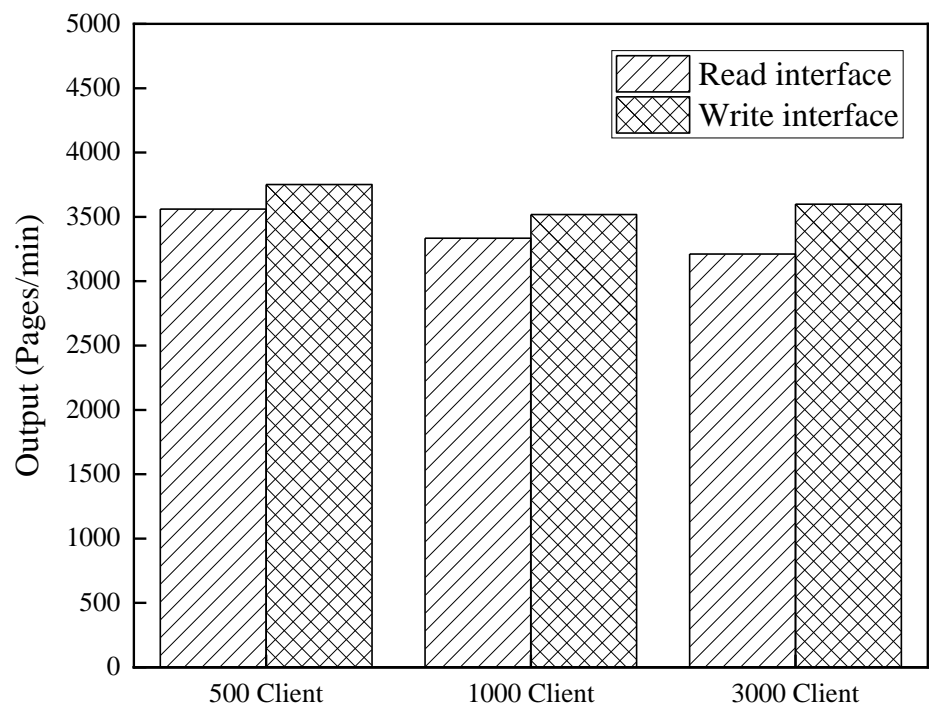

Fig. 5. Server stress test

\subsection{Test results of function modules of education information management platform}

The test results show that in the intelligent education platform, all functions can be used normally, and users can successfully complete the operations of registration, login, educational resources storage and sharing, remote classroom and so on. Administrator can $\log$ in to the system successfully to realize personnel management and service configuration operation. During the test, users can enter the platform and complete a series of operations from different clients such as personal computer, mobile phone and tablet. The educational resources storage, remote classroom function and educational administration management are introduced in detail.

Educational resources storage: In this function module, users can upload, download and share educational resources, common file formats such as PDF, WORD, TXT are supported, the initial upload space of each person is 500G, and subsequent expansion is supported. Administrators can enter the background system to query and manage the whole resource space and the usage of each user intelligently. Figure 6 shows the specific situation. 


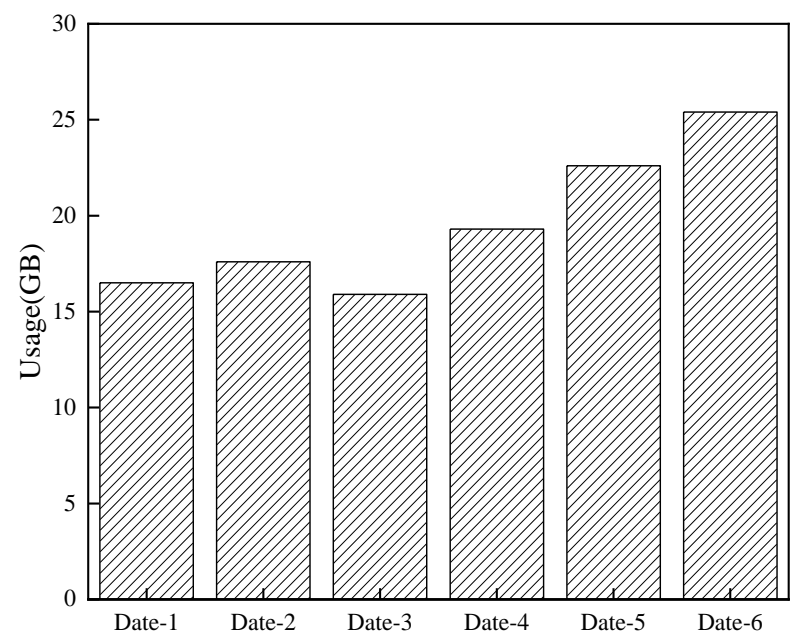

Fig. 6. Test of educational resource storage function

The administrator enters the management system to query the space usage of 6 users. Six users use 16.5GB, 17.6GB, 15.9GB, 19.3GB, 22.6GB and 25.4GB, respectively. The test results show that the educational resource storage function of the platform is well implemented and meets the expected demand.

Remote classroom function: Remote classroom is mainly carried out in the form of live broadcast. Teachers need to $\log$ in to the e-class, explain the content to students through the screen sharing function of computer, and complete the class content by using electronic courseware and PPT. At the same time, the remote classroom function also has the function of online examination. The teacher publishes the test questions at a specific time and publishes the correct answers in the background. Students will enter the system to answer questions, and the answers will be uploaded in real time. Then, the teacher marks the paper and publishes the test results. As shown in Figure 7, score query is taken as an example. Teachers can query the scores of 10 students through the platform. The platform can clearly display the scores of the 10 students and show the scores of different types of questions such as multiple-choice questions, judgment questions, and blank filling questions, so that teachers can adjust the class content according to the scores of different types of questions to further improve the teaching quality. The test results show that the remote classroom function runs well and meets the design needs. 


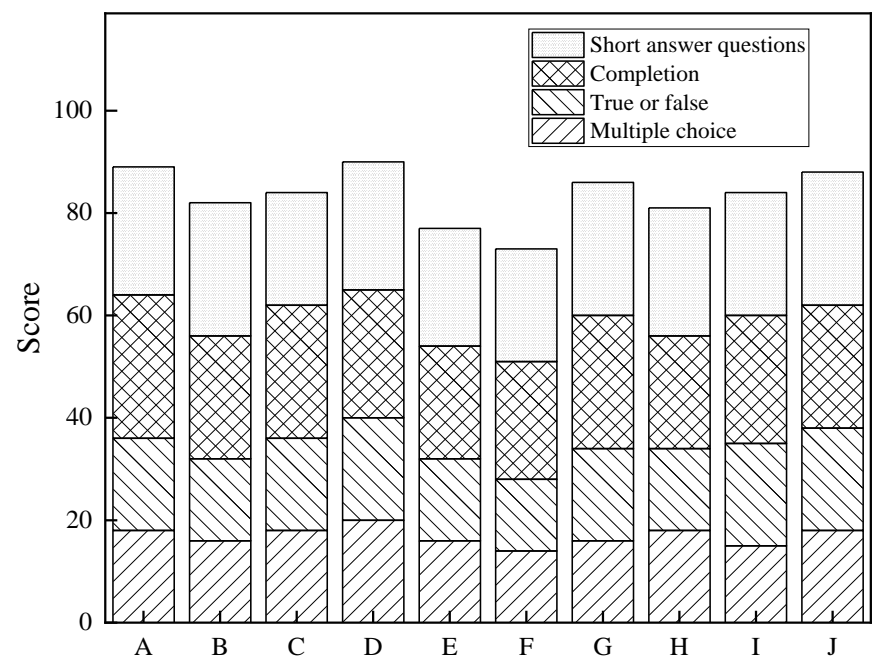

Fig. 7. Function test of score query in remote classroom

Educational administration management function: Education management platform links teachers, students and school administrators, and has the functions of information retrieval, classroom management, score input, score query and so on. Student information query management is taken as an example. Managers can query the information of student A through the platform. Table 3 shows the specific situation of student A.

Table 3. Function test of student information query in educational administration management

\begin{tabular}{|l|c|l|c|}
\hline \multicolumn{1}{|c|}{ Category } & \multicolumn{1}{c|}{ Content } & \multicolumn{1}{c|}{ Category } & Content \\
\hline Name & A & Gender & 1802 \\
\hline student ID & 2018120211502 & class & 2018 \\
\hline grade & 4 & Start year & network engineering \\
\hline College & School of Information Science & profession & \\
\hline
\end{tabular}

The platform can be used to clearly query the basic information of the student, greatly improving the work efficiency of school administrators. Moreover, the information can be modified in real time, which further improves the reliability and effectiveness of the data. The test results show that the educational administration management function runs smoothly and meets the design requirements. 


\section{Conclusion}

Present problems existing in school management are fully considered. Based on AI and cloud computing technology, a set of intelligent education platform suitable for daily management of colleges and universities is designed. The platform is divided into three parts: the bottom facilities layer, the system construction layer and the application experiment layer. It effectively serves the students, teachers and administrators, and realizes the two core functional areas of intelligent education service and intelligent education application. The related detection results of the platform show that when the million level data is inserted, the total time consumption of the sequential read and write in the database of the platform is $752 \mathrm{~s}$ and $312 \mathrm{~s}$, respectively, with the rate of $1330 \mathrm{row} / \mathrm{s}$ and $3205 \mathrm{row} / \mathrm{s}$, respectively; the total time consumption of random read and write in the database is $968 \mathrm{~s}$ and $211 \mathrm{~s}$, respectively, with the rates of 1033 row $/ \mathrm{s}$ and 4739 row $/ \mathrm{s}$, respectively, indicating that the database of the platform can support large-scale data access. In $60 \mathrm{~s}$, when the number of clients is 500,1000 and 3000 , the average rates of read interface and write interface are 3560 pages/min and $3752 \mathrm{pages} / \mathrm{min} ; 3334 \mathrm{pages} / \mathrm{min}$ and $3518 \mathrm{pages} / \mathrm{min} ; 3211 \mathrm{pages} / \mathrm{min}$ and 3598 pages/min, respectively, which indicates that the system of the platform can still run stably under high concurrency. The test results of platform function module show that users can enter the platform and complete a series of operations from different clients such as personal computer, mobile phone and tablet. The platform has stable and orderly operation of educational resources storage, remote classroom function and educational administration management function, which meets the expected functional requirements.

\section{$5 \quad$ References}

[1] Ali M, Khan S U, Vasilakos A V. (2015). Security in cloud computing: Opportunities and challenges. Information ences, 305:357-383. https://doi.org/10.1016/j.ins.2015.01.025

[2] González-Martínez, José A, Bote-Lorenzo M L, Gómez-Sánchez, Eduardo, et al. (2015). Cloud computing and education: A state-of-the-art survey. Computers \& Education, 80:132-151. https://doi.org/10.1016/j.compedu.2014.08.017

[3] Wen X W, Wang F L, Xing T. (2015). Application of Cloud computing to the education management information construction. Journal of Heilongjiang Institute of Technology, (3):59-62.

[4] Sabi H M, Uzoka F M E, Langmia K, et al. (2016). Conceptualizing a model for adoption of cloud computing in education. International Journal of Information Management, 36(2):183-191. https://doi.org/10.1016/j.ijinfomgt.2015.11.010

[5] Liu Y H, Chen J G, Zhang C Y. (2016). The scheme design of university teaching management plantform based on cloud computing. Journal of Changchun Normal University, 35(6):54-59.

[6] Peters, R. A. (2015). Anchored learning and the development of creative, critical thinking and life-long learning skills. Teaching Public Administration, 33(3):221-240. https://doi. org/10.1177/0144739415581077

[7] Hsu L, Martin R L, Mcelroy B, et al. (2015). Data management, sharing, and reuse in experimental geomorphology: Challenges, strategies, and scientific opportunities. Geomorphology, 244(sep.1):180-189. https://doi.org/10.1016/j.geomorph.2015.03.039 
[8] Capa-Aydin, Yesim. (2016). Student evaluation of instruction: comparison between inclass and online methods. Assessment \& Evaluation in Higher Education, 41(1):1-15. https ://doi.org/10.1080/02602938.2014.987106

[9] Davis H, Graham C. (2018). Navigating a career in tertiary education management in an era of unceasing transformation. Journal of Higher Education Policy \& Management, 40(2):97-106. https://doi.org/10.1080/1360080x.2018.1428932

[10] Li Z, Shen H, Ligon W, et al. (2016). An Exploration of Designing a Hybrid Scale-Up/Out Hadoop Architecture Based on Performance Measurements. IEEE Transactions on Parallel and Distributed Systems, 28(2):386-400. https://doi.org/10.1109/tpds.2016.2573820

[11] Elhoseny H, Elhoseny M, Abdelrazek S, et al. (2016). Utilizing Service Oriented Architecture (SOA) in Smart Cities. International Journal of Advancements in Computing Technology, 8(3):77-84.

[12] Bhandari G P, Ratneshwer. (2017). Fault Repairing Strategy Selector for Service-Oriented Architecture. International Journal of Modern Education \& Competence, 9(6):32-39. https ://doi.org/10.5815/ijmecs.2017.06.05

[13] Liu Q F. (2017). Design and Implementation of Wisdom Education Cloud Platform, Beijing University of Technology.

[14] Tan W, Fan Y, Ghoneim A, et al. (2016). From the Service-Oriented Architecture to the Web API Economy. IEEE internet computing, 20(4):64-68. https://doi.org/10.1109/mic.20 $\underline{16.74}$

[15] Goiri I, Bianchini R, Nagarakatte S, et al. (2015). Approx. Hadoop: Bringing Approximations to MapReduce Frameworks. Computer architecture news, 43(1):383-397. https://doi.org/10.1145/2786763.2694351

[16] Sharma Y, Verma S, Kumar S, et al. (2017). A Context-Based Performance Enhancement Algorithm for Columnar Storage in MapReduce with Hive. International Journal of Cloud Applications \& Computing, 3(4):38. https://doi.org/10.4018/ijcac.2013100104

[17] Veiga J, Exposito R R, Taboada G L, et al. (2016). Flame-MR: An event-driven architecture for MapReduce applications. Future Generation Computer Systems, 65(DEC.):46-56. https://doi.org/10.1016/i.future.2016.06.006

[18] Yuanzhao G, Binglong L I. (2017). A Forensic Method for Efficient File Extraction in HDFS Based on Three-Level Mapping. Wuhan University Journal of Natural Sciences, (22):126. https://doi.org/10.1007/s11859-017-1224-7

[19] Sharma Y, Verma S, Kumar S, et al. (2017). A Context-Based Performance Enhancement Algorithm for Columnar Storage in MapReduce with Hive[J]. International Journal of Cloud Applications \& Computing, 3(4):38. https://doi.org/10.4018/ijcac.2013100104

[20] Qi L H, Ai M H, Wang J H. (2014). Study of Hadoop Based Power Quality Monitoring Cloud Model. Electric Power Information and Communication Technology, 12(2):10-14.

\section{Author}

Qiang Li was born in Henan, China in 1982. From 2002 to 2006, he studied in Chongqing University and received his bachelor's degree in 2006. From 2007 to 2010, he studied in Chongqing University and received her Master's degree in 2010. He has published 5 papers. His research interests are included digital media design and artificial intelligence. Email: levisBJ@163.com

Article submitted 2020-12-05. Resubmitted 2021-01-11. Final acceptance 2021-01-12. Final version published as submitted by the authors. 